\title{
Direct Comparison of Standard and Ultrasensitive PCR for the Detection of Plasmodium falciparum from Dried Blood Spots in Bagamoyo, Tanzania
}

\author{
Christine F. Markwalter, ${ }^{1}$ Billy Ngasala, ${ }^{2}$ Tonelia Mowatt, ${ }^{1}$ Christopher Basham, ${ }^{3}$ Zackary Park,${ }^{3}$ Mwajabu Loya, ${ }^{2}$ Meredith Muller, ${ }^{3}$ \\ Christopher Plowe, ${ }^{4}$ Myaing Nyunt, ${ }^{4}$ and Jessica T. Lin ${ }^{3 *}$ \\ ${ }^{1}$ Duke Global Health Institute, Duke University, Durham, North Carolina; ${ }^{2}$ Department of Parasitology and Medical Entomology, Muhimbili \\ University of Health and Allied Sciences, Dar es Salaam, Tanzania; ${ }^{3}$ Institute of Global Health and Infectious Diseases, University of North Carolina \\ School of Medicine, Chapel Hill, North Carolina; ${ }^{4}$ University of Maryland School of Medicine, Baltimore, Maryland
}

\begin{abstract}
Ultrasensitive PCR used in low-transmission malaria-endemic settings has revealed a much higher burden of asymptomatic infections than that detected by rapid diagnostic tests (RDTs) or standard PCR, but there is limited evidence as to whether this is the case in higher transmission settings. Using dried blood spots (DBS) collected among 319 schoolchildren in Bagamoyo, Tanzania, we found good correlation (Pearson's $R=0.995$ ) between Plasmodium falciparum parasite densities detected by a DNA-based 18s rRNA real-time PCR (qPCR) and an RNA-based ultrasensitive reverse transcriptase (RT)-PCR (usPCR) for the same target. Whereas prevalence by usPCR was higher than that found by qPCR (37\% versus 32\%), the proportion of additionally detected low-density infections (median parasite density $<0.050$ parasites/ $\mu \mathrm{L}$ ) represented an incremental increase. It remains unclear to what extent these low-density infections may contribute to the infectious reservoir in different malaria transmission settings.
\end{abstract}

Molecular assays have the potential to reveal the true burden of asymptomatic infection in malaria-endemic settings but usually require additional expertise and specific sample collection requirements. For this reason, comparisons of PCR and ultrasensitive PCR (usPCR) detection of malaria in endemic settings are not common and have mostly been limited to low-burden settings pursuing malaria elimination. In such settings, the asymptomatic reservoir as detected by ultrasensitive assays far outweighs cases detectable by conventional rapid diagnostic tests (RDTs) or even standard PCR. For example, sensitive loop-mediated isothermal amplification and RT-qPCR assays deployed in southwest Ethiopia detected a roughly $7 \times$ greater $P$. falciparum prevalence versus RDT, ${ }^{1}$ whereas ultrasensitive assays implemented in a preelimination setting in South Africa detected 3.9\% prevalence in a cohort that was completely negative by RDT and nested PCR. ${ }^{2}$

It is unclear whether the same degree of excess low-density infections exists in higher transmission settings and whether ultrasensitive assays are needed to capture the infectious reservoir in its entirety in these settings. In this study, we used easily collected dried blood spots (DBS) to compare detection of $P$. falciparum by a standard DNA-based real-time PCR (qPCR) with an usPCR that targets 18s rRNA transcripts. qPCR was implemented at our field site in Bagamoyo, Tanzania, whereas the usPCR was later performed in a U.S. research laboratory from concurrently collected DBS.

Blood samples were collected from rural Bagamoyo district in eastern Tanzania, where malaria transmission is moderate but declining. Malaria screening was conducted from October to November 2018 at four primary schools in Bagamoyo district (Fukayosi, Mtakuja, Mkenge, and Mwavi) as part of a malaria transmission study. We targeted asymptomatic persons $>5$ years of age for screening-defined as those without fever, negative report of fevers or chills in the previous 3 days,

\footnotetext{
*Address correspondence to Jessica T. Lin, Division of Infectious Diseases, University of North Carolina School of Medicine, 130 Mason Farm Rd., Suite 2115, Chapel Hill, NC 27599. E-mail: jessica_lin@ med.unc.edu
}

and negative report of antimalarial use within the previous 7 days. The median age of those screened during this time period was 10 years (IQR: 8-12 years). Males and females were equally represented ( $53 \%$ and $47 \%$, respectively).

Approximately $200 \mu \mathrm{L}$ of finger-prick blood was used to simultaneously make thick and thin blood smears, perform a dual antigen histidine-rich protein 2 (HRP2) and pLDH RDT (SD Bioline, Abbott, Columbus, OH), and create 2-3 DBS on Whatman $3 \mathrm{MM}$ filter paper, each with $50 \mu \mathrm{L}$ of blood. Rapid diagnostic tests were read as positive for $P$. falciparum if the HRP2 band was present. For qPCR analysis, the entirety of a single $50 \mu \mathrm{L}$ blood spot was punched into deep well plates and extracted using Chelex resin after overnight incubation in 10\% saponin. Extracted DNA was subjected to real-time PCR targeting $18 \mathrm{~s}$ rRNA as previously described, ${ }^{3}$ but modified for use with Sahara Hot Start Master Mix (Chai Biotechnologies Inc, Santa Clara, CA). Duplicate reactions were carried out in portable real-time PCR instruments (Open qPCR, Chai Biotechnologies) situated in a renovated laboratory adjacent to Bagamoyo District Hospital. A sample was considered positive if a single reaction of two was positive with a $\mathrm{Ct}$ value $<40$ cycles. Parasite densities were quantified based on standards generated from mock blood spots created using 3D7 cultured parasites spiked into whole blood. A human B-tubulin qPCR assay was used to verify adequate DNA extraction in all $P$. falciparumpositive samples and a subset of negative samples. ${ }^{4}$ The limit of detection of this qPCR deployed at the field site has been determined to be 1 parasite $/ \mu \mathrm{L}(95 \% \mathrm{Cl} 0.7,2.3 \mathrm{p} / \mu \mathrm{L})$.

Dried blood spot samples collected in Bagamoyo were stored at room temperature and then shipped to the United States, where they were stored at $4^{\circ} \mathrm{C}$. Approximately 9 months after collection, usPCR was performed as previously described. ${ }^{5,6}$ This multiplex assay amplifies and detects total nucleic acids (DNA and RNA transcripts) of the $P$. falciparum and Plasmodium vivax 18s rRNA genes. As parasites are estimated to express $10^{3}-10^{4}$ transcripts per parasite, ${ }^{7}$ the addition of reverse transcription leads to a highly sensitive assay. Previous studies reported detection limits of 0.016 parasites/ $\mu \mathrm{L}$ when performed from $50 \mu \mathrm{L}$ DBS samples. ${ }^{6}$ Samples were 


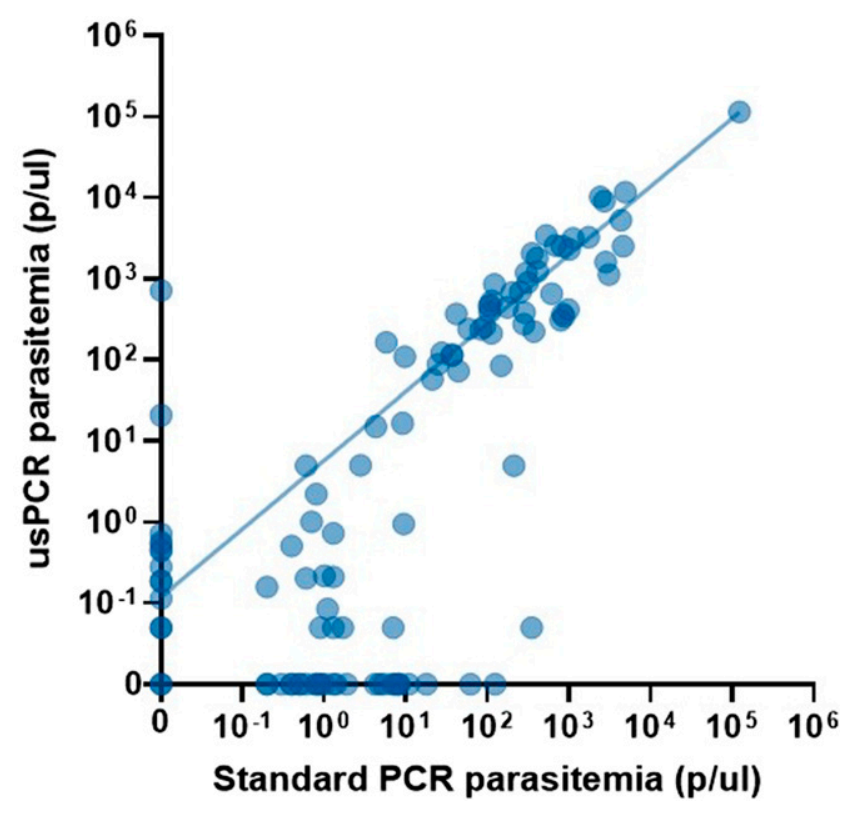

FIGURE 1. Plasmodium falciparum parasite densities determined by standard PCR and usPCR were in agreement (slope: 0.844 , Pearson's $R$ : 0.995). Note: Error bars (SD) are smaller than symbol size. This figure appears in color at www.ajtmh.org.

selected for usPCR if three total DBS were originally collected (roughly $60 \%$ of samples).

Extraction and amplification for usPCR were successful in all DBS samples based on amplification of human actin as an internal control. In addition, as expected, usPCR consistently detected 0.005 parasites $/ \mu \mathrm{L}$ in $50 \mu \mathrm{LDBS}$ standards made from $P$. falciparum culture-spiked whole blood. The assay was performed in duplicate, and samples were considered positive only if two runs had Ct values $<37$. If duplicates were discordant, a third usPCR reaction was performed as a "tie breaker." Parasite densities were determined based on $50 \mu \mathrm{L}$ DBS standard curves. $P$. vivax was not detected in any samples.

Plasmodium falciparum parasite densities in the cohort as determined by qPCR and usPCR were strongly correlated, especially at higher parasite densities. The slope of the linear regression was $0.844(0.829,0.859)$, close to the expected value of one (Pearson's $R$ 0.995) (Figure 1). This suggests that, despite transport and storage time, 18s rRNA transcripts were relatively stable and remained detectable from DBS at expected levels.
As expected, the measured prevalence of malaria increased with improved analytical assay sensitivity (Figure 2A). Parasite prevalence by microscopy was 14\% (44/319). Using conventional RDTs, which typically have detection limits around 100 parasites/ $\mu \mathrm{L}, 20 \%$ (63/319) of individuals were malaria positive. By GPCR, malaria prevalence was significantly greater at $32 \%(102 / 319)$, whereas $37 \%$ (118/319) of individuals were malaria positive by usPCR. Thus, usPCR detected almost twice as many infections as RDT, with a more incremental increase from standard PCR.

Our finding supports the idea that as malaria prevalence increases, the proportion of additionally detected ultralowdensity infections seems to diminish, similar to patterns seen in other studies ${ }^{8,9}$ (Table 1). In Rufiji, Tanzania, where light microscopy prevalence was $25 \%$, the detection of lowdensity parasitemias was double by qPCR (50\%), but only incrementally more by usPCR $(58 \%) .{ }^{10}$ Similarly, in a Papua New Guinea cohort, where $P$. falciparum qPCR prevalence was $29 \%$, the multi-target var ATS usPCR detected incrementally more infections (33\%), whereas the high-volume multi-target usPCR detected a prevalence of $53 \%$, still not quite double the qPCR prevalence. ${ }^{11}$ By contrast, in lower burden settings, the reservoir of ultralow-density parasitemias unmasked by usPCR is many times greater than that readily identified by RDT or microscopy ${ }^{1,2}$ (Table 1).

Concordance of $P$. falciparum detection among RDT, qPCR, and usPCR mostly aligned with expectation based on test characteristics (Figure 2A). All RDT-positive samples were also positive by usPCR. There were a number of qPCRpositive samples that were usPCR negative $(n=33)$. If stringency for qPCR positivity is increased and requires both duplicates to have $\mathrm{Ct}<40$, then only 15 samples fall into this category. This discrepancy between the two methods is probably explained by low-level RNA degradation during sample transport, storage, or extraction. In total, usPCR detected an additional 49 malaria-positive individuals compared with qPCR. The median parasite density for samples detectable only by usPCR was very low: $<0.05$ parasites $/ \mu \mathrm{L}$ or 50 parasites/mL (IQR: $<0.05-<0.05$ parasites $/ \mu \mathrm{L}$ ) (Figure $2 \mathrm{C}$ ).

Regions where malaria is commonplace are expected to have a higher number of false-positive RDTs arising from recent treatment, due to persistence of HRP2 at detectable levels within the circulation for weeks after treatment. ${ }^{13}$ Indeed, seven samples were RDT positive but negative by microscopy and qPCR. These same samples were positive by usPCR as well. We have observed that $18 \mathrm{~S}$ rRNA transcripts
A

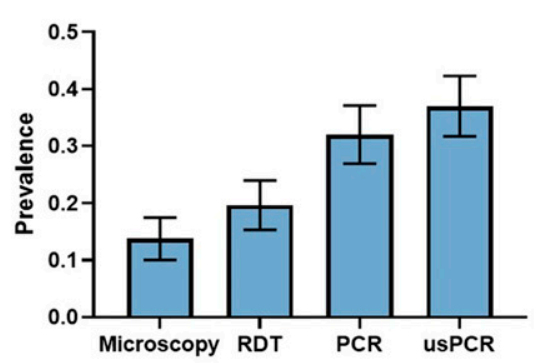

B $\mathrm{N}=\mathbf{3 1 9}$

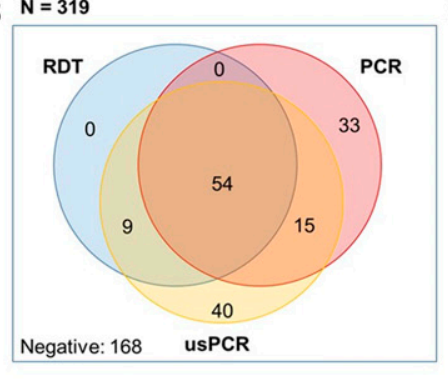

C

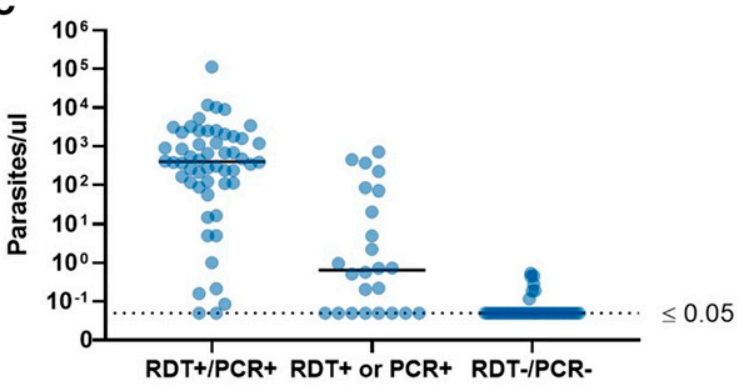

FIGURE 2. (A) Prevalence measured by microscopy, rapid diagnostic test (RDT), PCR, and usPCR. (B) Agreement Venn diagram among the RDT, PCR, and usPCR methods. (C) Parasite densities of usPCR + samples by assay positivity. Number of samples in each category: 54 (RDT+/PCR+), 24 (RDT + or PCR+), and 40 (RDT-/PCR-). This figure appears in color at www.ajtmh.org. 
TABLE 1

The proportion of additionally detected infections by ultrasensitive molecular methods is associated with transmission intensity

\begin{tabular}{|c|c|c|c|c|c|c|}
\hline \multirow[b]{3}{*}{ Reference } & \multirow[b]{3}{*}{ Setting } & \multirow[b]{3}{*}{$N$} & \multicolumn{3}{|c|}{ Plasmodium falciparum prevalence by detection method, \% } & \multirow[b]{3}{*}{ Ratio } \\
\hline & & & Microscopy/RDT & Standard $\mathrm{PCR}^{\star}$ & usPCR & \\
\hline & & & 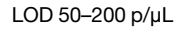 & LOD: $0.1-10 \mathrm{p} / \mu \mathrm{L}$ & LOD: $0.002-0.2 \mathrm{p} / \mu \mathrm{L}$ & \\
\hline Awandu et al. ${ }^{2}$ & South Africa & 1,475 & 0 & 0 & 3.9 & $\mathrm{~N} / \mathrm{A}$ \\
\hline Gruenberg et al. ${ }^{9}$ & Brazil & 651 & - & 0.80 & 1.7 & $-: 1: 2.1$ \\
\hline Gruenberg et al. $^{9}$ & Thailand & 773 & - & 1.3 & 1.9 & -: $1: 1.5$ \\
\hline Gruenberg et al. $^{9}$ & Papua New Guinea & 828 & - & 8.6 & 12.2 & $-: 1: 1.4$ \\
\hline Girma et al. ${ }^{1}$ & Gambella region, Ethiopia & 562 & $2.0 \dagger$ & $13 \ddagger$ & 21.5 & $0.15: 1: 1.7$ \\
\hline Hofmann et al. ${ }^{11}$ & Madang Province, Papua New Guinea & 300 & 8.1 & 29 & $\begin{array}{c}33 \\
53 \text { (high-volume) }\end{array}$ & $0.28: 1: 1.1$ \\
\hline This work & Bagamoyo, Tanzania & 319 & $17 \dagger$ & 32 & 37 & $0.53: 1: 1.2$ \\
\hline Hofmann et al. ${ }^{10}$ & Rufiji, Tanzania & 498 & 25 & 50 & 58 & $0.50: 1: 1.2$ \\
\hline
\end{tabular}

are detectable up to 2 weeks after successful treatment (unpublished), likely expressed by circulating gametocytes present at low levels after treatment. ${ }^{14,15}$ In further support of this possibility, we additionally tested DBS from three subjects who were RDT positive but qPCR negative for both asexual parasites (18s rRNA) and gametocytes (Pfs25 and PF3D7_0630000 assays), yet were infectious to mosquitoes. ${ }^{16}$ Two reported ACT treatment within the previous 28 days. We found that all were usPCR positive, suggesting persistent HRP2 antigen may coincide with ultralow-level gametocytemia posttreatment. Thus, a fraction of the surfeit of purported very low-density parasitemias $(7 / 49$ or $14 \%$ here) may actually represent previous infection or posttreatment gametocytemia in settings where malaria and its treatment are commonplace.

Unfortunately, the contribution of very low-density infections detectable only by usPCR to malaria transmission in settings like Bagamoyo remains unknown. In theory, even very low-density infections have the potential to become higher density infections; the transmission potential of these infections probably depends on their chronicity and dynamics, as well as how frequently they generate transmissible gametocyte densities. ${ }^{17-20}$ Within this sample set, a subset of 27 subjects were enrolled into a malaria transmission study and thus evaluated using gametocyte-specific RT-PCR assays within days of malaria screening. Twenty of 27 samples were positive for gametocytes, with $19 / 20$ positive by $18 \mathrm{~s}$ rRNA qPCR. This suggests that usPCR may provide diminishing returns with regard to identifying the infectious reservoir, but the sample size was small, and RDT-/PCR-/usPCR + subjects were not included. Ongoing, longitudinal mosquito skin feeding assays and gametocyte assays will reveal the extent to which asymptomatic low-density infections contribute to the infectious reservoir in this setting. ${ }^{16}$

Received September 19, 2020. Accepted for publication December 29, 2020.

Published online February 8, 2021.

Acknowledgments: We thank the study participants, school teachers, as well as the Tanzanian field staff who made the study possible. This work was supported by the NIH Grant R01Al137395 (to J. T. L.), the North Carolina Translational and Clinical Sciences (NC TraCS) Institute (UL1TR002489), NIH Grant U19AI129386 (to M. M. N. and C. V. P.), the Bill \& Melinda Gates Foundation (OPP1171753), and the Duke Global Health Institute.
Authors' addresses: Christine F. Markwalter and Tonelia Mowatt, Duke Global Health Institute, Duke University, Durham, NC, E-mails: christine.markwalter@duke.edu and toneliamowatt@gmail.com. Billy Ngasala and Mwajabu Loya, Department of Parasitology and Medical Entomology, Muhimbili University of Health and Allied Sciences, Dar es Salaam, Tanzania, E-mails: bngasala70@yahoo.co.uk and loyamwajabu51@gmail.com. Christopher Basham, Zackary Park, Meredith Muller, and Jessica T. Lin, Division of Infectious Diseases, University of North Carolina School of Medicine, Chapel Hill, NC, E-mails: christopher_basham@med.unc.edu, zackarypark@gmail.com, meredith_muller@med.unc.edu, and jessica_lin@med.unc.edu. Christopher Plowe and Myaing Nyunt, Department of Medicine, University of Maryland School of Medicine, Baltimore, MD, E-mails: plowe.chris@ gmail.com and myaingnyunt@gmail.com.

\section{REFERENCES}

1. Girma S, Cheaveau J, Mohon AN, Marasinghe D, Legese R, Balasingam N, Abera A, Feleke SM, Golassa L, Pillai DR, 2019. Prevalence and epidemiological characteristics of asymptomatic malaria based on ultrasensitive diagnostics: a crosssectional study. Clin Infect Dis 69: 1003-1010.

2. Awandu SS, Raman J, Bousema T, Birkholtz LM, 2019. Ultralowdensity Plasmodium falciparum infections in African settings. Clin Infect Dis 69: 1463-1464.

3. Taylor SM, Juliano JJ, Trottman PA, Griffin JB, Landis SH, Kitsa P, Tshefu AK, Meshnick SR, 2010. High-throughput pooling and real-time PCR-based strategy for malaria detection. J Clin Microbiol 48: 512-519.

4. Beshir KB, Hallett RL, Eziefula AC, Bailey R, Watson J, Wright SG, Chiodini PL, Polley SD, Sutherland CJ, 2010. Measuring the efficacy of anti-malarial drugs in vivo: quantitative PCR measurement of parasite clearance. Malar J 9: 312.

5. Adams $M$ et al., 2015. An ultrasensitive reverse transcription polymerase chain reaction assay to detect asymptomatic lowdensity Plasmodium falciparum and Plasmodium vivax infections in small volume blood samples. Malar J 14: 520.

6. Zainabadi K, Adams M, Han ZY, Lwin HW, Han KT, Ouattara A, Thura S, Plowe CV, Nyunt MM, 2017. A novel method for extracting nucleic acids from dried blood spots for ultrasensitive detection of low-density Plasmodium falciparum and Plasmodium vivax infections. Malar J 16: 377.

7. Murphy SC et al., 2012. Real-time quantitative reverse transcription PCR for monitoring of blood-stage Plasmodium falciparum infections in malaria human challenge trials. Am J Trop Med Hyg 86: 383-394.

8. Bousema T, Okell L, Felger I, Drakeley C, 2014. Asymptomatic malaria infections: detectability, transmissibility and public health relevance. Nat Rev Microbiol 12: 833-840. 
9. Gruenberg M et al., 2020. Utility of ultra-sensitive qPCR to detect Plasmodium falciparum and Plasmodium vivax infections under different transmission intensities. Malar J 19: 319.

10. Hofmann N, Mwingira F, Shekalaghe S, Robinson LJ, Mueller I, Felger I, 2015. Ultra-sensitive detection of Plasmodium falciparum by amplification of multi-copy subtelomeric targets. PLoS Med 12: e1001788.

11. Hofmann NE et al., 2018. Assessment of ultra-sensitive malaria diagnosis versus standard molecular diagnostics for malaria elimination: an in-depth molecular community cross-sectional study. Lancet Infect Dis 18: 1108-1116.

12. Golassa L, Cheaveau J, Mohon AN, Pillai DR, 2019. Reply to Awandu et al. Clin Infect Dis 69: 1464-1465.

13. Dalrymple U, Arambepola R, Gething PW, Cameron E, 2018. How long do rapid diagnostic tests remain positive after anti-malarial treatment? Malar J 17: 228.

14. Hanron AE et al., 2017. Multiplex, DNase-free one-step reverse transcription PCR for Plasmodium 18S rRNA and spliced gametocyte-specific mRNAs. Malar J 16: 208.
15. Niederwieser I, Felger I, Beck HP, 2000. Plasmodium falciparum: expression of gametocyte-specific genes in monolayer cultures and malaria-positive blood samples. Exp Parasitol 95: 163-169.

16. Ngasala B et al., 2019. Detection of the Asymptomatic Plasmodium Falciparum Infectious Reservoir among Schoolchildren in Tanzania using Mosquito Skin Feeding Assays. Poster Presentation at the Annual Meeting of the American Society of Tropical Medicine and Hygiene, National Harbor, MD.

17. Lin JT, Saunders DL, Meshnick SR, 2014. The role of submicroscopic parasitemia in malaria transmission: what is the evidence? Trends Parasitol 30: 183-190.

18. Stone W, Gonçalves BP, Bousema T, Drakeley C, 2015. Assessing the infectious reservoir of falciparum malaria: past and future. Trends Parasitol 31: 287-296.

19. Slater $\mathrm{HC}$ et al., 2019. The temporal dynamics and infectiousness of subpatent Plasmodium falciparum infections in relation to parasite density. Nat Commun 10: 1433.

20. Imwong M et al., 2016. Numerical distributions of parasite densities during asymptomatic malaria. J Infect Dis 213: 1322-1329. 